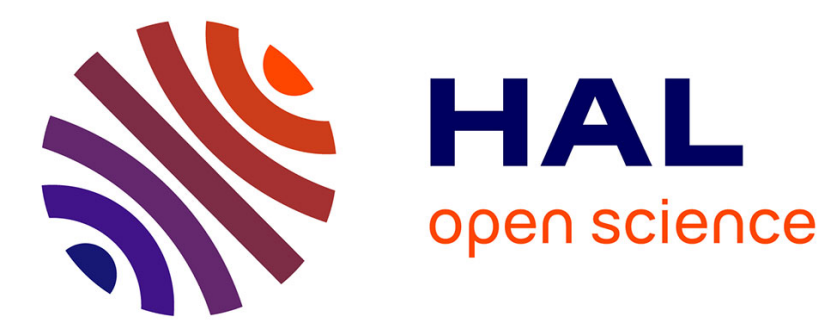

\title{
Modelling mesoporous alumina microstructure with 3D random models of platelets
}

\author{
Haisheng Wang, Andrea Pietrasanta, Dominique Jeulin, François Willot, \\ Matthieu Faessel, Loïc Sorbier, Maxime Moreaud
}

\section{- To cite this version:}

Haisheng Wang, Andrea Pietrasanta, Dominique Jeulin, François Willot, Matthieu Faessel, et al.. Modelling mesoporous alumina microstructure with 3D random models of platelets. Journal of Microscopy, 2015, 260 (3), pp.287-301. 10.1111/jmi.12295 . hal-01170616

HAL Id: hal-01170616

https://hal-mines-paristech.archives-ouvertes.fr/hal-01170616

Submitted on 1 Jul 2015

HAL is a multi-disciplinary open access archive for the deposit and dissemination of scientific research documents, whether they are published or not. The documents may come from teaching and research institutions in France or abroad, or from public or private research centers.
L'archive ouverte pluridisciplinaire HAL, est destinée au dépôt et à la diffusion de documents scientifiques de niveau recherche, publiés ou non, émanant des établissements d'enseignement et de recherche français ou étrangers, des laboratoires publics ou privés. 


\title{
Modelling mesoporous alumina microstructure with 3D random models of platelets
}

\author{
Haisheng Wang ${ }^{1}$, Andrea Pietrasanta ${ }^{1}$, Dominique Jeulin ${ }^{1}$, François Willot*1, \\ Matthieu Faessel $^{1}$, Loïc Sorbier ${ }^{2}$ and Maxime Moreaud ${ }^{2}$ \\ ${ }^{1}$ MINES ParisTech, PSL Research university, CMM - Centre for mathematical \\ morphology, 35, rue St Honoré, F-77300 Fontainebleau, France \\ ${ }^{2}$ IFP Energies nouvelles, Rond-point de l'échangeur de Solaize, BP 3, 69360 \\ Solaize, France
}

\begin{abstract}
This work focuses on a mesoporous material made of nanometric alumina "platelets" of unknown shape. We develop a 3D random microstructure to model the porous material, based on 2D Transmission Electron Microscopy (TEM) images, without prior knowledge on the spatial distribution of alumina inside the material. The TEM images, acquired on samples with thickness $300 \mathrm{~nm}$, a scale much larger than the platelets's size, are too blurry and noisy to allow one to distinguish platelets or platelets aggregates individually. In a first step, the TEM images correlation function and integral range are estimated. The presence of long-range fluctuations, due to the TEM inhomogeneous detection, is detected and corrected by filtering. The corrected correlation function is used as a morphological descriptor for the model. After testing a Boolean model of platelets, a two-scales model of microstructure is introduced to replicate the statistical dispersion of platelets observed on TEM images. Accordingly a set of two-scales Boolean models with varying physically-admissible platelets shapes is proposed. Upon optimization, the model takes into account the dispersion of platelets in the microstructure as observed on TEM images. Comparing it to X-ray diffraction and nitrogen porosimetry data, the model is found to be in good agreement with the material in terms of specific surface area.
\end{abstract}

Keywords: Mesoporous alumina; transmission electron microscopy; image analysis; random models; specific surface;

\footnotetext{
${ }^{*}$ Corresponding author. E-mail: francois.willot@mines-paristech.fr, Telephone: 01646948 07, Fax: 0164694707 .
} 


\section{Introduction}

Mesoporous alumina is a class of porous material made-up by an assemblage of alumina $\left(\mathrm{Al}_{2} \mathrm{O}_{3}\right)$ grains at the nanometric scale and is widely used in industry as catalyst supports (Misra, 1987). The support of a catalyst provides reaction interface to the catalysts and reagents by porosity. The catalytic performances and more generally transport properties of mesoporous materials are strongly influenced by the morphology of the porous phase, including the porosity, specific surface area and connectivity properties (Levitz, 1993; Wernert et al., 2010). This influence of the morphology of the support is experimentally observed for activity (Khodakov et al., 2002; Rana et al., 2011), selectivity (Khodakov et al., 2002) or deactivation (Prieto et al., 2014) for important industrial process such as methanol synthesis (Prieto et al., 2014), FischerTropsch synthesis (Khodakov et al., 2002) or hydrotreating of heavy oil fractions (Rana et al., 2011). The preparation method and synthesis conditions, especially temperature and type of solvent influence the morphology of the alumina porous structure at the nanoscale (Chiche et al., 2008; Trimm \& Stanislaus, 1986). A fine description of the microstructure is required to predict and optimize the adsorption and catalytic performance of these materials.

Different characterization techniques allow one to extract information about the inner microstructure or on the spatial dispersion of grains at the nanoscale. These techniques include nitrogen porosimetry, X-ray diffraction (XRD), transmission electron microscopy (TEM) and tomography (Kim et al., 2003; Roiban, 2010). Nitrogen porosimetry provides information about the porosity at the macro-scale. XRD methods provide information of the mean structuring crystallites and on morphological characteristics such as size and shape, less on pore connectivity or structure at higher lengthscale (Chiche et al., 2008). TEM methods produce transmission 2D images on a thin slice of material, from which 3D reconstruction is difficult. Electron tomographic techniques may provide 3D images of the pore space at the nanoscale but at the expense of long acquisition and reconstruction time and at poor representativeness (volume sides limited to a few hundreds of nanometers).

Morphological modelling is a key procedure in the design, development and optimization of catalyst supports. On the one hand, simple models are created using deterministic microstructures such as periodic arrays (Mu et al., 2008). On the other hand, more elaborate modelling relies on stochastic microstructures to mimic real materials (Adler, 1994; Diaz et al., 2004; Kočí et al., 2006). With the reconstructed microstructures, simulations of adsorption (Štěpanék et al., 2007) have been performed, as well as heat conduction (Kohout et al., 2004) and reaction-diffusion processes (Kočí et al., 2007).

To compensate for the lack of available information, assumptions are required to reconstruct the microstructure in 3D from 2D images. Said otherwise, a 3D microstructure model which results in the observed TEM images, but also respect physical assumptions (on e.g. crystallography for the grains shape) is sought for. This modelling problem is solved by means of numerical or (semi-)analytical optimization (Couka et al., 2015; Jean et al., 2010; Jeulin, 2012). Stochastic random media with parameters estimated by optimization are simulated accordingly. Ideally, the models incorporate 
all morphological information provided by characterization techniques (Moreaud et al., 2012).

In this work, information is first extracted on a mesoporous alumina sample using nitrogen adsorption porosimetry and TEM images (Sec. 2). The textural fluctuations at different scales and dispersion phenomena are studied by means of the representative volume element method, and the correlation function (Secs. 3). Secondly, one-scale and multiscale random models of mesoporous alumina are developed and compared (Secs. 4 and 5). They take into account the shape and the spatial dispersion of grains. The models are optimized to reproduce the correlation function observed on TEM images, while using alumina of varying physically-admissible grain sizes. Finally, some properties of the simulated models are compared with available nitrogen porosimetry data (Sec. 6).

\section{Experimental characterization}

The mesoporous alumina sample is obtained from a thermal treatment of a commercial boehmite powder $\left(350 \mathrm{~m}^{2} / \mathrm{g}\right.$, provided by Axens, Salindres, France). The commercial powder is dispersed in water to obtain a $10 \mathrm{wt} \%$ solution. The suspension is stirred for 2 hours and put in an autoclave. The autoclave is sealed and heated at $150^{\circ} \mathrm{C}$ for 7 hours. The suspension is filtered and dried at $120^{\circ} \mathrm{C}$ overnight. The powder is shaped in trilobed extrudates, the extrudates are dried at $80^{\circ} \mathrm{C}$ overnight and calcined under dry air $\left(650^{\circ} \mathrm{C}, 4 h\right)$ then wet air $\left(700^{\circ} \mathrm{C}, 2 h, 6 w t \%\right.$ water $)$. The obtained calcined extrudates are translucent, indicating no scattering by light, that is to say, negligible density fluctuation at the scale of visible light wavelength (few hundred nanometers).

Specific surface area $S_{B E T}$ (in $\mathrm{m}^{2} / \mathrm{g}$ ) is measured by nitrogen adsorption with the Brunauer-Emmett-Teller (BET) method (Brunauer et al., 1938). Extrudates are pretreated at $350^{\circ} \mathrm{C}$ for $3 h$ under vacuum $\left(10^{-4} \mathrm{~Pa}\right)$ before measurement. Relative uncertainty of the obtained specific surface area is estimated to be $5 \%$. Structural density $d_{s}$ (in $\mathrm{g} / \mathrm{cm}^{3}$ ) is measured by $\mathrm{He}$ Pycnometry. The sample is pretreated at $250^{\circ} \mathrm{C}$ for $3 \mathrm{~h}$. Expected relative uncertainty is about $0.5 \%$. Grain density $d_{g}$ (in $g / \mathrm{cm}^{3}$ ) is measured by mercury intrusion after a pretreatment $\left(250^{\circ} \mathrm{C}\right.$ for $\left.2 h\right)$. The grain density is obtained at a $0.2 \mathrm{MPa}$ intrusion pressure where mercury fills only inter-grain porosity but not intra-grain porosity. Expected relative uncertainty is $2.5 \%$ (Tab. 1).

The porosity $\varepsilon$ (void volume fraction) is obtained from :

$$
\varepsilon=1-\frac{d_{g}}{d_{s}}
$$

Specific surface area $S_{V}$ in $m^{-1}$ is obtained by :

$$
S_{V}=10^{6} \frac{S_{B E T}}{d_{g}}
$$

Relative uncertainties for $\varepsilon$ and $S_{V}$ are then respectively $3.6 \%$ and $5.6 \%$. More details are shown in table 1. 


\begin{tabular}{|l||r|r|}
\hline & Value & Absolute uncertainty \\
\hline$d_{g}\left(\mathrm{~g} / \mathrm{cm}^{3}\right)$ & 1.04 & 0.026 \\
\hline$d_{s}\left(\mathrm{~g} / \mathrm{cm}^{3}\right)$ & 3.33 & 0.017 \\
\hline$S_{B E T}\left(\mathrm{~m}^{2} / \mathrm{g}\right)$ & 240 & 12 \\
\hline$\varepsilon$ & 0.688 & 0.025 \\
\hline$S_{V}\left(\mathrm{~nm}^{-1}\right)$ & 0.231 & 0.0129 \\
\hline
\end{tabular}

Table 1: Textural properties and their uncertainties obtained from the porosimetry measurements.

The shape and size of alumina crystals at the nanometric scale, however, remains unknown. In this work, a morphological model for the platelets of a similar material (Chiche et al., 2008), consisting of prisms is used with irregular octagonal basis (Fig. 1). The basis is made up of three sides with lengths $D_{1}, D_{2}$ and $D_{3}$. The angles, equal to $127.8^{\circ}$ and $270-127.8=142.2^{\circ}$, are fixed by crystallography. With the prism's height $D_{4}$, the platelets shape is parametrized by $\left(D_{i}\right)_{1 \leq i \leq 4}$. X-ray diffraction data for the oxide nanoparticles (Chiche et al., 2008) indicate $D_{1}=3.4 \mathrm{~nm}$, $D_{2}=1.4 \mathrm{~nm}, D_{3}=0.4 \mathrm{~nm}$ and $D_{4}=3.7 \mathrm{~nm}$ (Fig. 1). These values do not necessarily correspond to the material we consider, so that we let $\left(D_{i}\right)_{1 \leq i \leq 4}$ vary.

Nevertheless, for consistency with XRD measurements, we fix the ratios $D_{2} / D_{1}=$ 0.41 and $D_{3} / D_{1}=0.12$, as obtained in (Chiche et al., 2008). With these constraints, the platelets shape are fully parametrized by the two variables $D_{1}$ and $D_{4}$. The work of Chiche (Chiche et al., 2008) indicates that materials synthesized in solvent of different $\mathrm{pH}$ values have different particle morphologies, in other words, varying shape of octagonal basis and varying width. For the sample we use in this work, the $\mathrm{pH}$ value is already known.

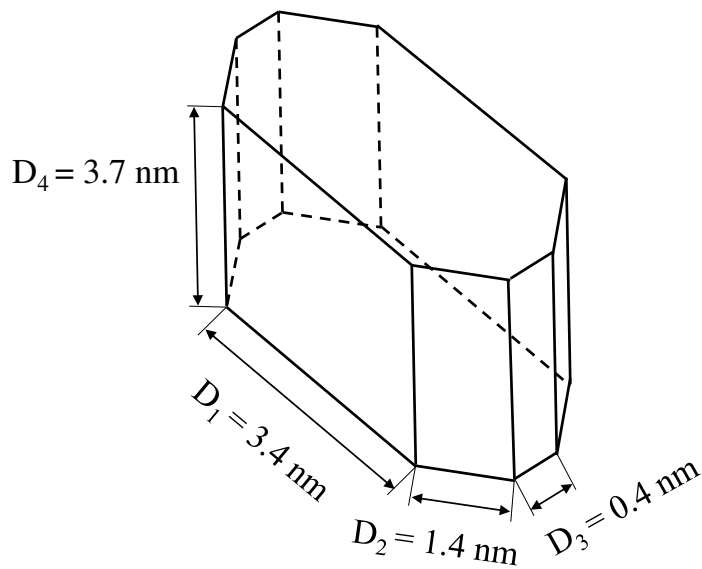

Figure 1: Alumina platelet shape, following (Chiche et al., 2008).

For TEM imaging, an extrudate has been put in an oven at $80^{\circ} \mathrm{C}$ overnight and embedded in araldite resin (EMbed 812, provided by Electron Microscopy Sciences, Hatfield, PA, USA). The included sample has been trimmed to obtain a trapezoidal surface 
and then cut in thin slices using an ultramicrotome (Reicherd). Slices thickness has been targeted to $300 \mathrm{~nm}$. Only slices with homogeneous color (homogeneous thickness) were withdrawn to a lacey carbon grid. Images were taken with a TEM (JEM-2100F, provided by $J E O L$, Peabody, MA, USA) operating at $200 \mathrm{kV}$, in bright field mode, using a CCD camera (UltraScan, provided by Gatan, Pleasanton, CA, USA). This camera has a four quadrant CCD captor and 2048 by 2048 pixels maximum resolution. Before taking the images, the gain and dark noise of the camera was calibrated respectively in a hole and in absence of electron beam. Images were taken at full resolution (2048 by 2048 pixels), with a $2 s$ exposure time at an indicated magnification of 15000 . This magnification yields a field of view of 1130 by $1130 \mathrm{~nm}^{2}$ and a pixel size of $0.552 \mathrm{~nm}$.

Twelve images were taken for different slices and different non overlapping zones were neither the edge of the slices, nor cutting artifacts, nor lacey carbon were apparent. One of them is represented in Fig. (2). Electrons are directed along the thickness of the samples and smallest dimension, normal to the surface layer, hereafter denoted by $\mathbf{e}_{3}$. We also introduce Cartesian axis $\mathbf{e}_{1}$ and $\mathbf{e}_{2}$ normal to $\mathbf{e}_{3}$ and parallel to the surface of the sample. Referring to the coordinates $x_{1}, x_{2}$ and $x_{3}$ in the Cartesian basis, 2D TEM images of porous media are modeled by the Lambert-Beer law (Reimer \& Kohl, 2008, p.36):

$$
Y\left(x_{1}, x_{2}\right)=a+b \exp \left[-c \int_{0}^{f} \mathrm{dx}_{3} \chi_{S}\left(x_{1}, x_{2}, x_{3}\right)\right],
$$

where $Y\left(x_{1}, x_{2}\right)$ is the greylevel TEM image at location $\left(x_{1}, x_{2}\right), f=300 \mathrm{~nm}$ is the sample thickness, $a$ and $b$ are constants related to the offset and gain of the camera, $c$ is the density function of the solid phase and $\chi_{S}$ is the characteristic function of the solid phase in $3 \mathrm{D}$, i.e. $\chi_{S}(x, y, z)=1$ if and only if point $(x, y, z)$ is in the solid (alumina) phase. Monte-Carlo simulations of $200 \mathrm{keV}$ electrons trajectories across alumina embedded in resin selected by a $120 \mu \mathrm{m}$ objective diaphragm gives a value $c=2.4010^{-3} \pm 2.0510^{-6} \mathrm{~nm}^{-1}$. Furthermore, the integral is bounded by $f$ and the sample has a high porosity $\varepsilon$, therefore the Eq. (1) is approximated by:

$$
Y\left(x_{1}, x_{2}\right) \approx(a+b)-b c \int_{0}^{f} \mathrm{dx}_{3} \chi_{S}\left(x_{1}, x_{2}, x_{3}\right) .
$$

The approximation is used for the simulation of TEM images from digital 3D microstructures afterwards. The histogram $P_{Y}$ of a 32-bit TEM image is represented in Fig. (3). Most of the values $Y\left(x_{1}, x_{2}\right)$ lie in-between $y_{1}=405$ and $y_{2}=660$. To visualize the TEM images, we threshold all values larger than $y_{2}$ or smaller than $y_{1}$, and normalize the result in the range $[0 ; 255]$. This amounts to replace $Y$ with:

$$
\widetilde{Y}=\frac{255}{y_{2}-y_{1}}\left(\max \left\{\min \left[Y\left(x_{1}, x_{2}\right), y_{2}\right], y_{1}\right\}-y_{1}\right)
$$

Local alignments and textured patterns are somehow visible in Fig. (2), but overall, information in the TEM images is hidden (and lost) by the attenuation of the electron beam along the samples's thickness. Values in the field's histogram (Fig. 3) does not allow one to segment the image into several zones of interest.

For electron tomography, few calcined extrudates were crushed in a mortar and the obtained powder was dispersed in ethanol. A drop of the suspension was put on a 


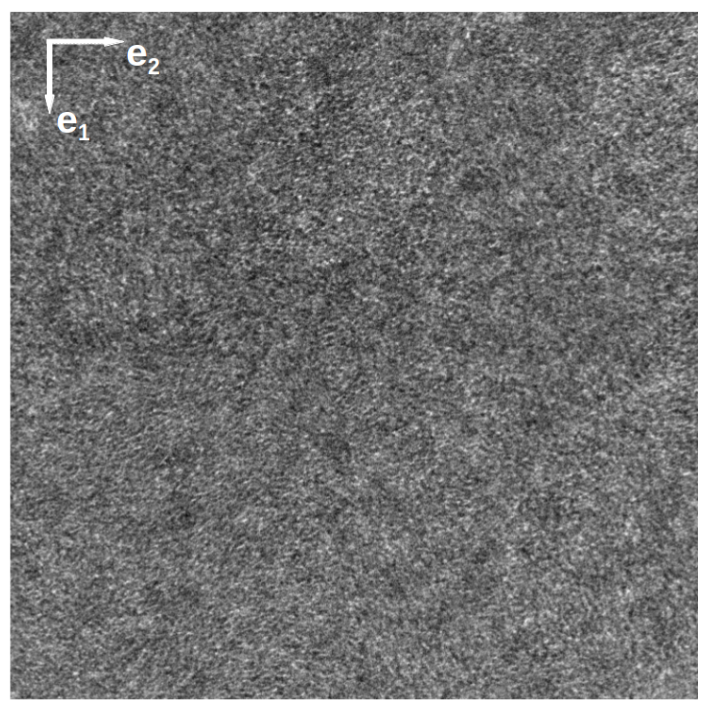

Figure 2: TEM image $\widetilde{Y}$ (see text) of mesoporous alumina $(1130 \mathrm{~nm} \times 1130 \mathrm{~nm})$.

holey carbon grid that was dried. Tilt series projections were acquired on the JEOL JEM-2100F fitted with the Gatan Ultrascan CCD camera, in bright field mode at $200 \mathrm{kV} .143$ projections with an indicated magnification of 40000 times $(0.21 \mathrm{~nm}$ pixel size) were acquired at full camera resolution (2048 by 2048 pixels). The 143 tilt angles range between $-71^{\circ}$ to $71^{\circ}$ with a Saxton spacing scheme. No fiducial marker was put on the grid to avoid the artifacts induced in their vicinity. Prior to reconstruction, images were binned twice to obtain a 1024 by 1024 pixels tilt series. The 3D volume was reconstructed with the robust method based on inverse problem approach to align marker-free projections and reconstruct 3D volume (Tran et al., 2014). A 3D view of the tomographic model is represented in Fig. (4).

\section{$3 \quad$ TEM image statistics}

In this section, two quantitative morphological types of information are extracted, the correlation (or covariance) function, which will be used in the morphological modelling, and the variance of the fields's local means, (Secs. 4 and 5).

\subsection{Correlation function}

Referring to two $2 \mathrm{D}$ vectors $\mathbf{x}=\left(x_{1} ; x_{2}\right)$ and $\mathbf{h}=\left(h_{1} ; h_{2}\right)$, the empirical correlation function of $Y$ is the 2D function estimated from the space average denoted by $\langle$.$\rangle :$

$$
C(\mathbf{h})=\frac{\langle Y(\mathbf{x}+\mathbf{h}) Y(\mathbf{x})\rangle-\langle Y(\mathbf{x}+\mathbf{h})\rangle\langle Y(\mathbf{x})\rangle}{\left\langle Y^{2}(\mathbf{x})\right\rangle-\langle Y(\mathbf{x})\rangle^{2}},
$$

so that $C(\mathbf{0})=1$ and $C(\infty)=0$ for a stationary field. Note that the correlation function does not depend on the values of $a, b$ and $c$ in (1) and that $C(\mathbf{h})$ in general 


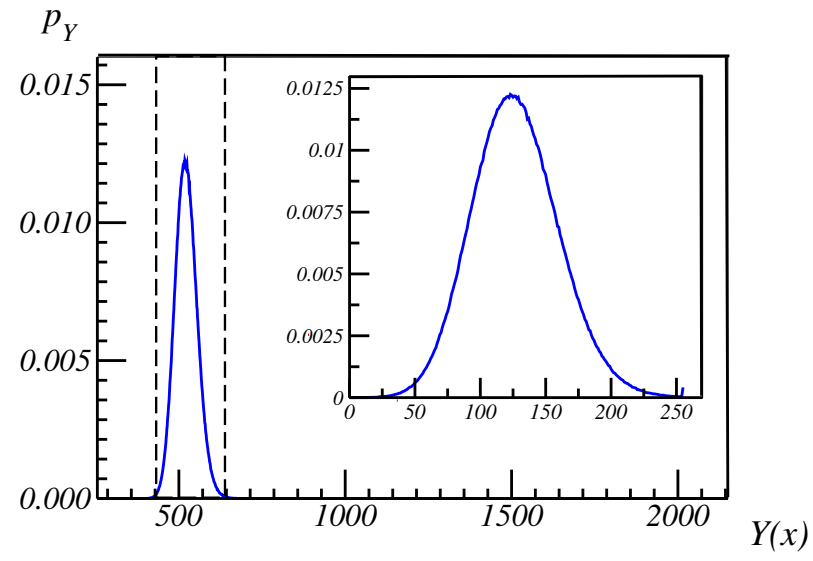

Figure 3: Histogram of the greylevels of the raw data in the range $[0 ; 2140]$. The vertical dotted lines are $Y(x)=y_{1,2}$. Top-right encrusted graph: histogram of $\widetilde{Y}$ (Eq. 3) in the range $[0 ; 255]$.

depends on the norm and orientation of $\mathbf{h}$. The function $C(h)$ in $(4)$ is computed using Fourier transforms (FFTs), which amounts to periodize the image. The profiles $C\left(h \mathbf{e}_{1,2}\right)$ along $\mathbf{e}_{1}$ and $\mathbf{e}_{2}$, for $h>0$, are represented in Fig. (5) for two TEM images. For the first sample, the two functions are sensibly different for intermediate values of $h \lesssim 6 \mathrm{~nm}$, underlying the image anisotropy. This anisotropy effect is smaller in other samples (Fig. 5b). Anisotropy effects are more easily apparent on the 2D representation $C\left(h_{1}, h_{2}\right)$ of the correlation function at long distance $h \gg 1$ (Fig. 6) shown in color scale. The figure is cropped with top-left corner's coordinate $(10 \mathrm{~nm}, 10 \mathrm{~nm})$, to avoid the high contrast when $h$ is small. Highest values are in red and lowest values in blue. Such results are not consistent with an isotropic distribution of platelets.

\subsection{Integral Range}

We follow (Jeulin, 2011; Kanit et al., 2003; Matheron, 1971) and define the variance $D^{2}(S)$ of the means $\langle Y\rangle_{S}$ of the function $Y$ over a compact subset of area (Lebesgue measure) $S$ by:

$$
D^{2}(S)=E\left\{\langle Y\rangle_{S}^{2}\right\}-\left(E\left\{\langle Y\rangle_{S}\right\}\right)^{2},
$$

In the above, $E\{\cdot\}$ is the expectation operator (estimated here by averaging). When the area approaches a point (or a pixel in $2 \mathrm{D}$ discrete image), we obtain the point variance $D_{1}^{2}$ :

$$
D_{1}^{2}=\left\langle Y^{2}\right\rangle-\langle Y\rangle^{2}
$$

The following asymptotic expansion holds (Lantuejoul, 1991; Matheron, 1971):

$$
D^{2}(S) \sim \frac{D_{1}^{2} A_{2}}{S},
$$

where:

$$
A_{2}=\int_{\mathcal{I}} \mathrm{d} \mathbf{h} C(\mathbf{h}),
$$




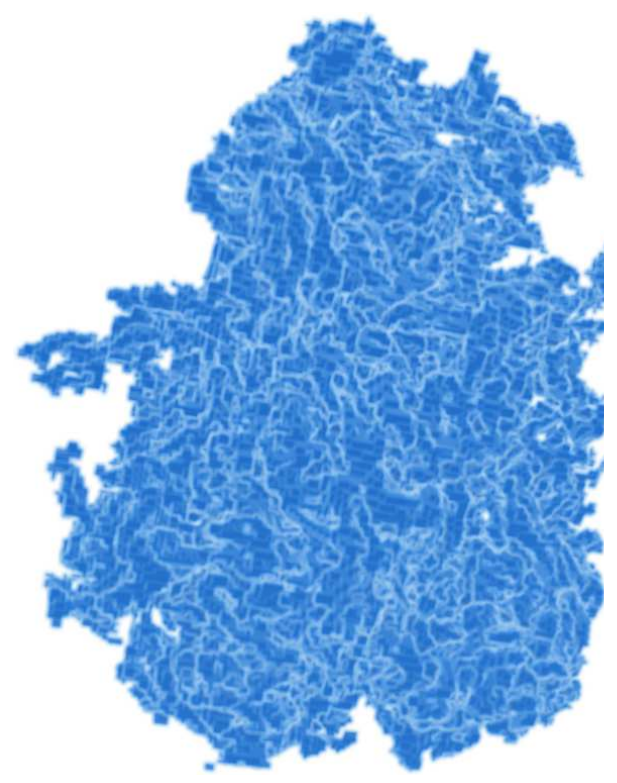

Figure 4: 3D view of the tomographic reconstruction of a small portion of mesoporous alumina. The image resolution is $0.66 \mathrm{~nm}^{3}$ per voxel and the image size is $328 \mathrm{~nm} \times 290 \mathrm{~nm} \times$ $257 \mathrm{~nm}$.

as long as $A_{2}$, the integral range over the support of the image $\mathcal{I}$, is finite. The integral range gives the typical surface area of the microstructure. In practice, use of the correlation function $C(h)$ in (8) can hardly be used to estimate $A_{2}$, due to fluctuations in the function $C(\mathbf{h})$ when $|\mathbf{h}|$ is large. Instead, the expansion (7) is generaly used (Altendorf et al., 2014), provided that numerical data follows the theoretical law (7). Hereafter, we check if this law is verified. The variances $D^{2}(S)$ are estimated at increasing values of $S$. This is done by dividing the TEM image into non-overlapping squares of equal area $S$, and measuring the variance of the mean over the subdomains (Eq. 5). The results are given in Fig. (7), in log-log scale for 5 images. An extra point representing $D^{2}(S)$ for $S=2048 \times 2048$ pixels is computed using all 12 images. The data follows the scaling law $D^{2}(S) \sim S^{-0.5}$, with an exponent less than 1, i.e. a much slower decrease than that in (7). Similar conclusions hold for the other 7 TEM images of the same sample. This is not consistent with the asymptotic result of Eq. (7), which is valid for any ergodic stationary random function with a finite integral range. The slowly-decreasing scaling law of the variance is explained by a drift in the image, as detailed below.

\subsection{Image drift}

To visualize variations in the images over large scales, we divide it into a set of nonoverlapping square subdomains of size $140 \mathrm{~nm} \times 140 \mathrm{~nm}$. The means over each square are represented in Fig. (8), for two random images. A drift appears on the two images, roughly oriented along the diagonal from the top-left to the bottom-right. This nonuniform averaged field can be interpreted as the result of a non-uniform detection of the camera or a non-constant thickness of the slices. Indeed, the four quadrants of the 


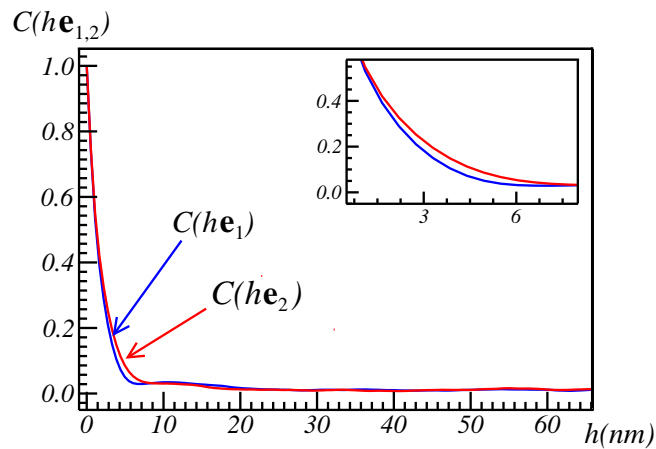

(a)

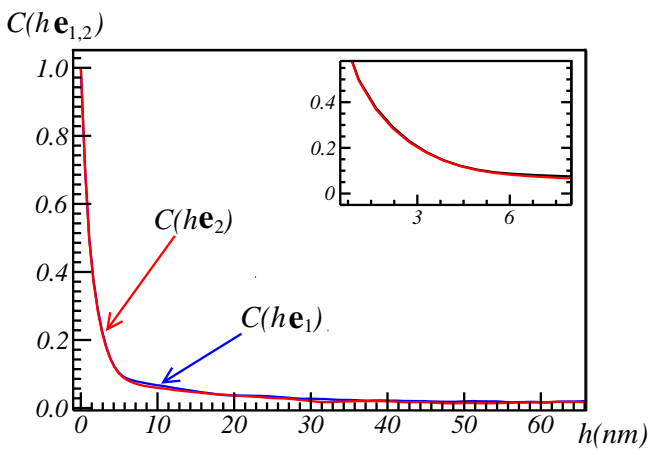

(b)

Figure 5: Correlation function profiles along the horizontal and vertical axis, for two TEM images ( $\mathrm{a}$ and $\mathrm{b})$.

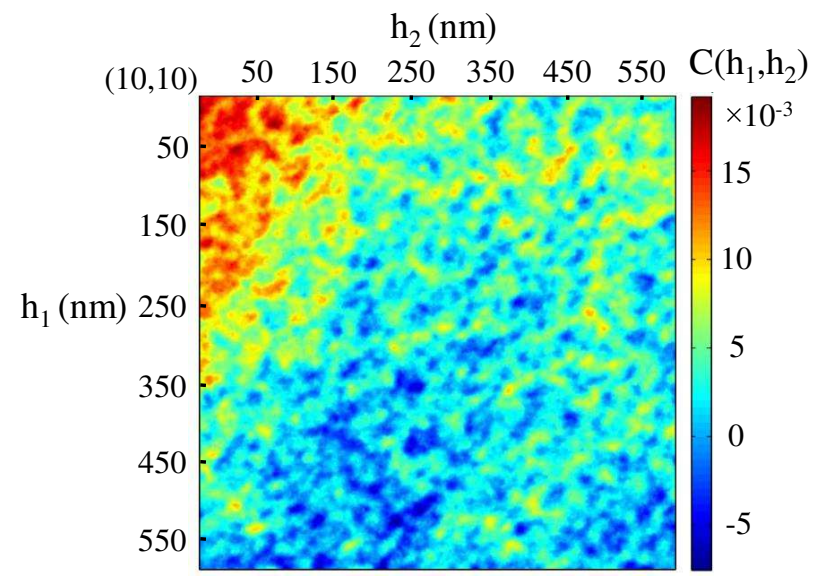

Figure 6: 2D correlation function $C\left(h_{1}, h_{2}\right)$ of the TEM image.

camera can be guessed from Fig. (8). This indicates that the correction of the gain of the camera performed before recording the images is not accurate enough to correct for the camera detection discrepancies. This is confirmed by the display of moving averages on more images of the sample, as shown in Fig. (9).

To remove the drift, a moving average is subtracted to each image, computed as the mean of a disk of radius $\ell$ centered on each pixel. Accordingly $Y$ is replaced by

$$
Y^{\prime}(\mathbf{x})=Y(\mathbf{x})-\frac{1}{\pi \ell^{2}} \int_{\left|\mathbf{x}-\mathbf{x}^{\prime}\right|<\ell} \mathrm{d} \mathbf{x}^{\prime} Y\left(\mathbf{x}-\mathbf{x}^{\prime}\right),
$$

where $\mathbf{x}=\left(x_{1}, x_{2}\right)$ is a point in the image. The value of $\ell$ is now chosen so that the integral range $A_{2}^{\prime}$ of the convoluted field becomes finite. Equivalently, $\ell$ is chosen so that the variance $D_{Y^{\prime}}^{2}(S)$ of the means over subdomains of size $S$ decreases with a scaling law $\sim 1 / S$, as in Eq. (7). We emphasize that this variance is computed on the corrected image $Y^{\prime}$, rather than on the moving average itself. We find numerically that $D_{Y^{\prime}}^{2}(S) \sim 1 / S^{\alpha}$ with $\alpha<1$ (resp. $\alpha>1$ ) when $\ell<110 \mathrm{~nm}$ (resp. $\ell>110$ $\mathrm{nm}$ ) and $D_{Y^{\prime}}^{2}(S) \sim 1 / S$ when $\ell \approx 110 \mathrm{~nm}$ (Fig. 10). Accordingly we set $\ell=110$ 


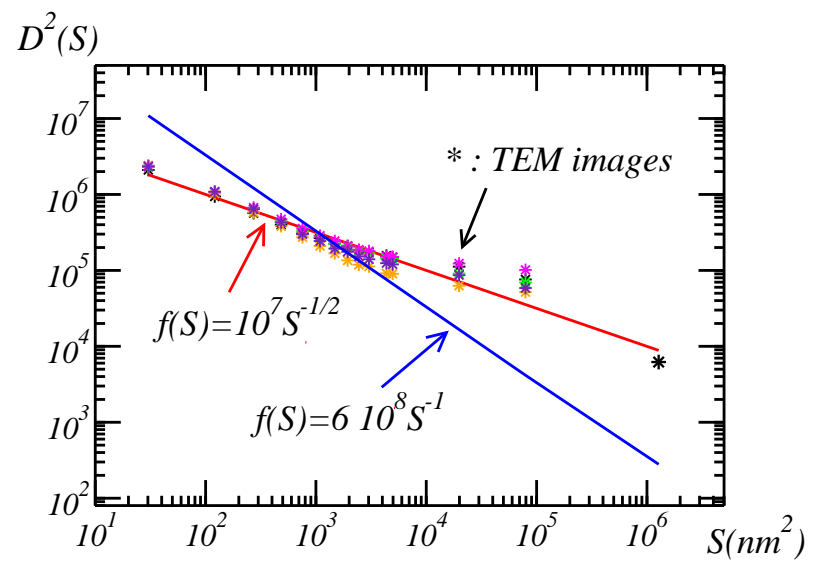

Figure 7: Variance $D^{2}(S)$ of the means over areas of surface $S$ in the image $Y$, computed using (5). Stars of different colors are used to distinguish between the 5 TEM images. Solid lines: numerical fit of the data $D^{2}(S) \sim S^{-0.5}$ (red), using all 5 samples, and, for comparison, scaling law $D^{2}(S) \sim 1 / S$ (blue) predicted by $(7)$.

nm and replace $Y$ with $Y^{\prime}$ in the rest of this study. The resulting moving average is represented in Fig. (9). Additionally the integral range of the 12 images is estimated, giving $A_{2}^{\prime} \approx 30 \mathrm{~nm}^{2}$.

After removing the drift, the long-range anisotropy observed in the correlation function almost disappears. Short-range anisotropy in the correlation function is also much smaller after drift-removing. In the following, it is approximated by an isotropic correlation function, denoted by $C_{T E M}(h)$, averaging over all directions and over the 12 TEM images.

\section{One-scale Boolean model}

To model the microstructure, we first consider a Boolean model of randomly-oriented platelets. We take first the octagonal prism presented in Sec. 2 as the shape of platelets. Microstructures are generated in two steps (Jeulin, 2000; Matheron, 1967):

- First, a realization of a Poisson random point process is generated with a prescribed point density, determined by the microstructure overall porosity, and the volume of the platelet.

- Second, an octagonal prism is translated at each Poisson point. The prism main axis orientation is uniform on the sphere.

This model should be considered as a one-scale model in the sense that it is based on a homogeneous Poisson point process. The Boolean model depends on the shape of the prisms and on the porosity. The latter pore volume fraction is set to $69 \%$, according to porosimetry data.

Realizations of the model are generated in a window consisting of $400 \times 400 \times 546$ voxels with resolution $0.55 \mathrm{~nm}$ per voxel. The upper surface of the microstructure 

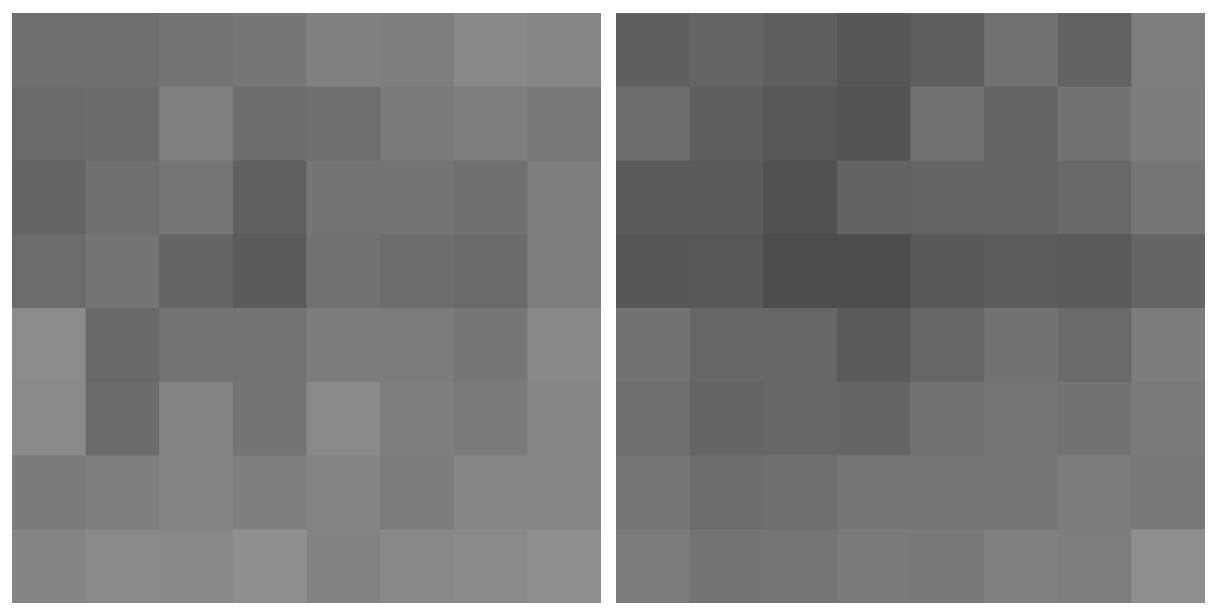

Figure 8: Local means computed over a set of non-overlapping square subdomains in two TEM images.
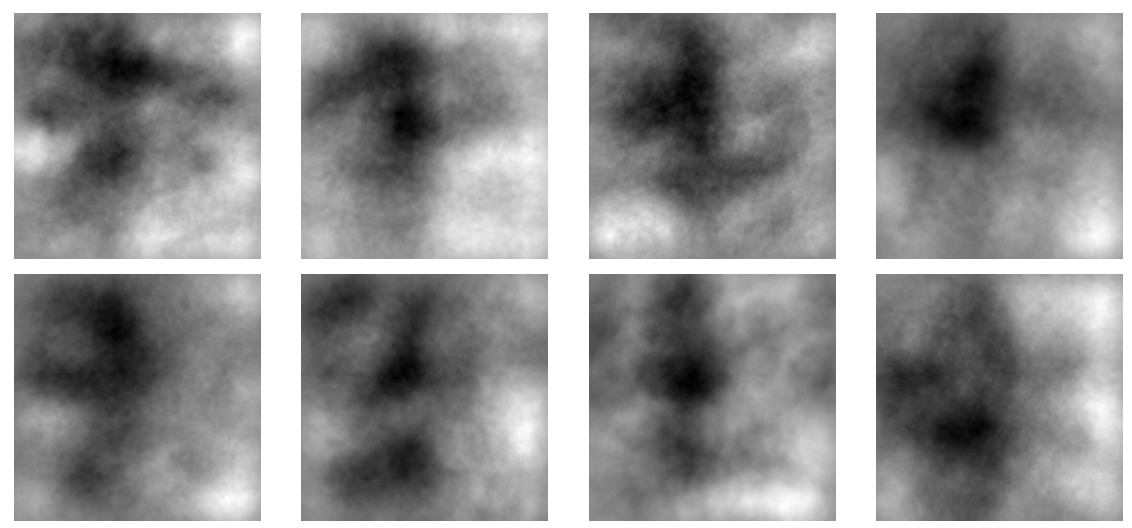

Figure 9: Moving average of 8 TEM images of the sample, using Eq. (9) with disk radius $110 \mathrm{~nm}$

$\left(\mathbf{e}_{1} ; \mathbf{e}_{2}\right)$ is discretized on a $400 \times 400$ voxels grid, and the thickness along $\mathbf{e}_{3}$ by 546 voxels. The microstructures have the same thickness as the sample material $(300 \mathrm{~nm})$. A $2 \mathrm{D}$ section of the $3 \mathrm{D}$ model is shown in Fig. (11). Transmission images in 2D are readily computed by performing an integral along the vertical axis $\left(\mathbf{e}_{3}\right)$ on the generated microstructure (Eq. 2). From the simulated transmission images, we obtain the corresponding $2 \mathrm{D}$ correlation function $C_{M}(\mathbf{h})$, who is confirmed to be isotropic. Its 1-D profile is hereafter denoted by $C_{M}(h)$. Since the correlation is normalized, the choice of the parameters $a, b$ and $c$ does not influence the estimation of correlation function. A comparison with the correlation functions of the TEM images $C_{T E M}(h)$ is represented in Fig. (12). 


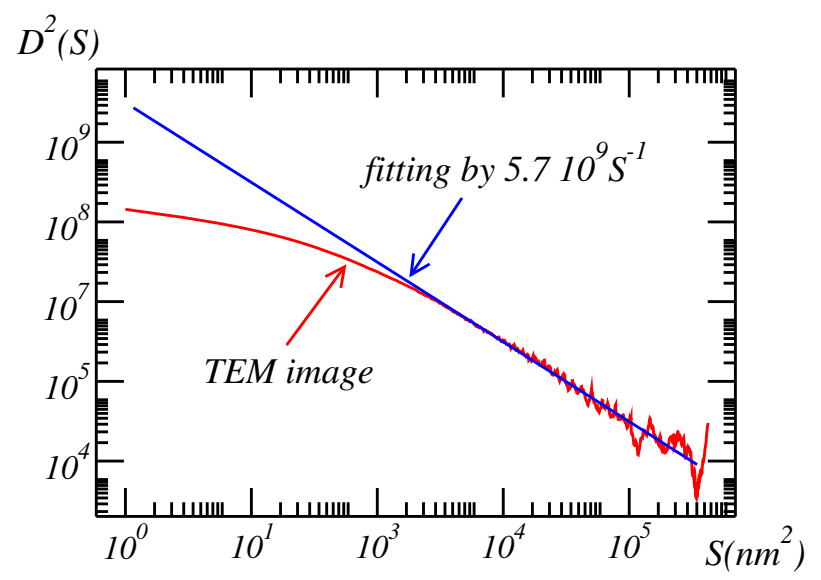

Figure 10: Variance $D_{Y^{\prime}}^{2}(S)$ of the means of image $Y^{\prime}$ over subdomains of area $S$, with respect to $S$ (red). Blue: numerical fit $D_{Y^{\prime}}^{2}(S) \sim 1 / S$ for large areas $S \gg A_{2}^{\prime}$.

The parameters of the one-scale Boolean model are estimated by minimizing some distance between the correlation functions measured on TEM and on simulated images:

$$
\inf _{M}\left\{\sum_{h=0}^{N} w_{h}\left[C_{T E M}(h)-C_{M}(h)\right]^{2}\right\},
$$

where $h$ is given in pixels units, $w_{h}$ are weights given by:

$$
w_{h}=\frac{a_{h}}{\sum_{i=0}^{N} a_{i}}, \quad a_{h}=\frac{1}{1+0.2 h},
$$

and $N=29$ defines a (finite) domain of comparison of the two correlation functions. The decreasing weights with $h$ are used to give more importance to the correlation function for small values of $h$, which is more reliable than when $h$ is large.

Previous findings (Chiche et al., 2008) indicate that the basis of the primary grain is octagonal. Under this assumption, the length $D_{1}$ and width $D_{4}$ of the octagonal grains vary independently, while the shape of the basis is fixed. Accordingly, the prism is parametrized by two variables. To examine the effects of both parameters on the correlation function, we fix one and let the other vary (Fig. 13). The slope at the origin $(h=0)$ of the correlation is driven by the platelet's shape: the thicker the platelet is ( $D_{4}$ increases), the smaller the slope of correlation will be. For an isotropic random set, it is related to the specific surface area $S_{V}$ (Matheron, 1967):

$$
\left.\frac{\mathrm{d} C_{M}(h)}{\mathrm{d} h}\right|_{h=0}=\frac{1}{4} S_{V}
$$

For a Boolean model with primary grain $A^{\prime}, S_{V}=-q \log (q) \frac{S_{A^{\prime}}}{V_{A^{\prime}}}$ where $q$ is the volume fraction of void, $S_{A^{\prime}}$ and $V_{A^{\prime}}$ are respectively the average surface area and average volume of primary grains. This can be used as an additional constraint to the optimization problem. Under this constraint, the width $D_{4}$ is fixed by the length $D_{1}$ or 


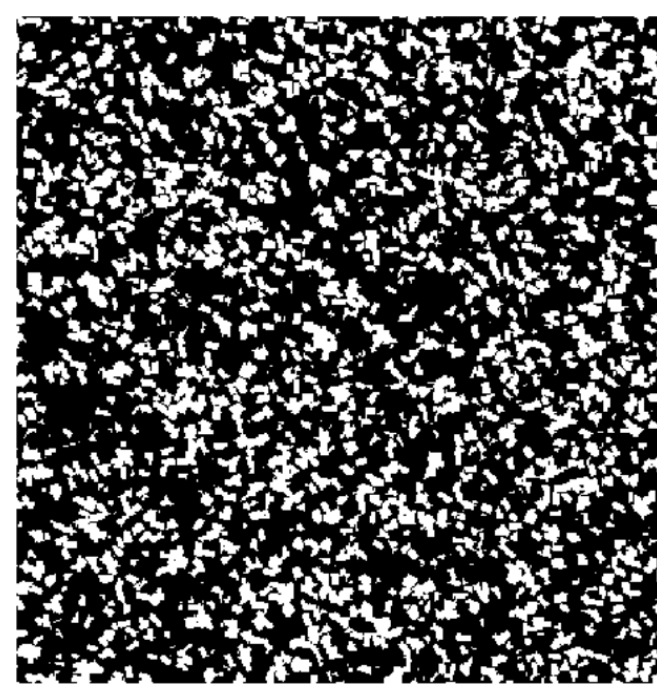

Figure 11: 2D section of a Boolean model of octagonal prisms. Solid phase in white.

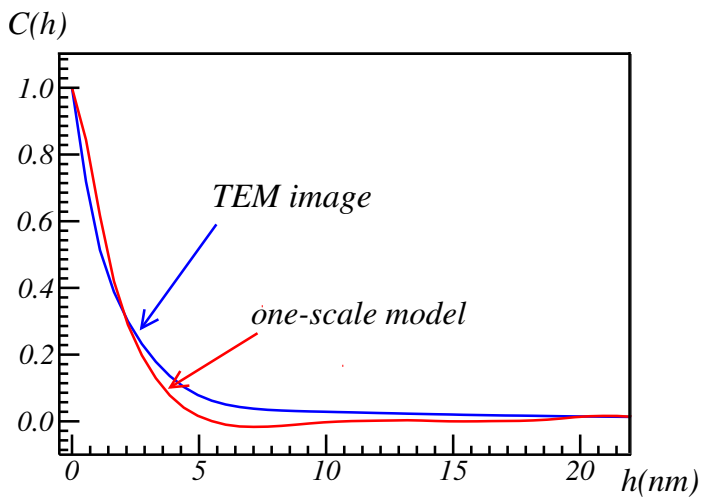

Figure 12: correlation of TEM images and of a computer-generated one-scale Boolean model. 
vice-versa. Therefore Boolean models following the additional constraint are generated and the resulting correlation functions are compared in Fig. (14). As expected, with the additional constraint, the models reproduce the correct slope at the origin for the correlation function, irrespective of the shape. However, the long-range of the correlation function is not reproduced except for very thin and highly elongated platelets (in red, in Fig. 14). These platelets shapes, with sizes smaller than $1 \mathrm{~nm}$, are hardly physical and must be rejected. Accordingly, models with larger correlation ranges must be used to approach the TEM images correlation function. Hereafter, a two-scales model is considered.

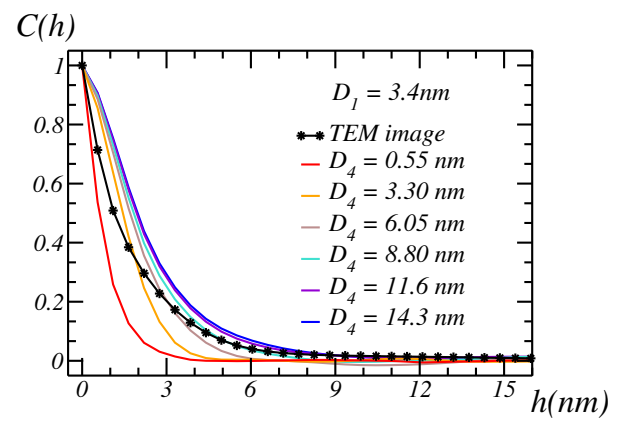

(a)

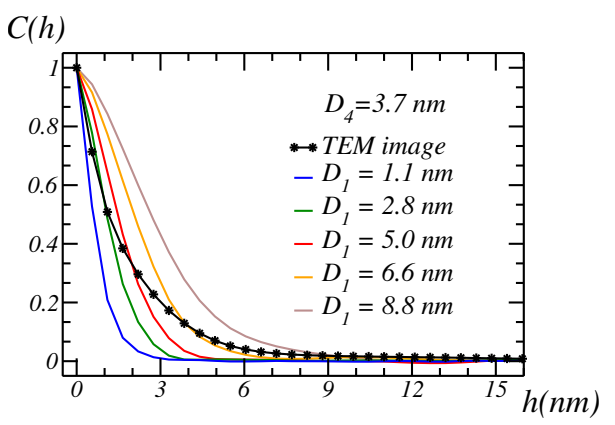

(b)

Figure 13: Effect of the grain shape on the correlation function of the one-scale model: with $D_{1}=3.4 \mathrm{~nm}$ and $D_{4}$ varying from $0.55 \mathrm{~nm}$ to $14.3 \mathrm{~nm}$ (a); with $D_{4}=3.7 \mathrm{~nm}$ and $D_{1}$ varying from $1.1 \mathrm{~nm}$ to $8.8 \mathrm{~nm}$ (b). The correlation function of the TEM images is shown in blue.

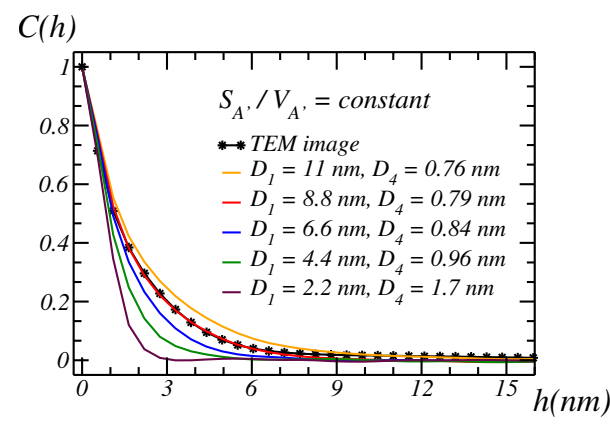

(a)

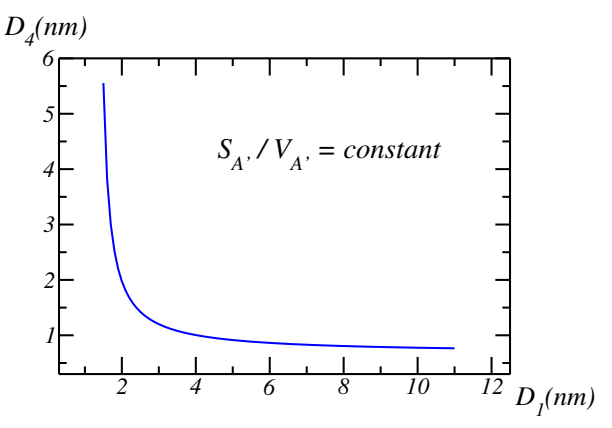

(b)

Figure 14: Correlation function of one-scale Boolean models of octagonal prisms with fixed surface density $S_{V}$ and increasing platelet width $D_{4}$ (a). Length $D_{1}$ with respect to width $D_{4}$ under the constraint in Eq. (12) (b).

\section{Two-scales Boolean model}

As previously seen, the one-scale model of random platelets can not reproduce the correlation function of the TEM images in the range larger than the size of the platelets. 


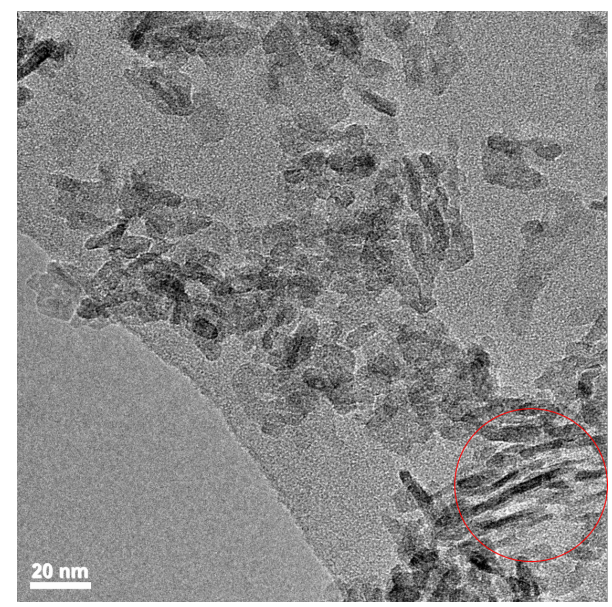

(a)

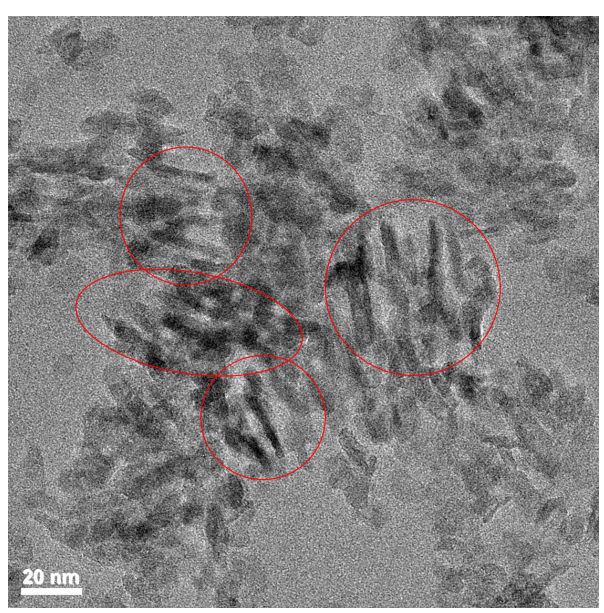

(b)

Figure 15: TEM image of crushed powder of mesoporous alumina. Local alignments are encircled in red.

Indeed, crushed powder of mesoporous alumina exhibits local alignments (Fig. 15). The aggregation of alumina platelets is well known (Euzen et al, 2002) and originates from the behavior of the boehmite precursor in solution (Fukusawa \& Tsujii, 1988). This aggregation is expected to be highly anisotropic due to the anisotropy of both platelet shape and the degree of hydroxylation of platelet faces (Digne et al., 2004). The alignments suggest the existence of at least one larger scale, which correspond to the size of aggregates of platelets. The size of these aggregates is in-between 2 to 3 times the size of platelets, according to (15). Thus, the local dispersion of the platelets should be taken into account.

Accordingly a simple two-scales Boolean model, more general than the previous one-scale model, is defined as follows:

- First, a Boolean model of spheres is used to generate a field of orientations: to each sphere is associated a random principal direction uniformly distributed on the unit sphere.

- Then, two Poisson point processes with two densities are generated inside and outside the spheres.

- Third, a platelet with fixed shape is located at each Poisson point. When the point is outside the spheres, the platelet orientation is uniformly random, as in the previous one-scale model. When the point is inside a sphere, the platelet orientation is given by that of the sphere. If a point is located in two or more spheres, one of the corresponding orientations is chosen at random.

This type of model is a variant of a Cox Boolean model (Jeulin, 2012). The twoscales Boolean model has 5 parameters: the shape parameters $D_{1}$ and $D_{4}$ (Fig. 1) and 3 other variables that control the spatial dispersion of platelets. The latter are the volume fraction of spheres at the larger scale $p_{S}$, the radius of the spheres $r_{S}$ and the volume fraction of platelets inside spheres $p_{A}$. The volume fraction of platelets outside 
the spheres $p_{S}^{\prime}$ is prescribed by the overall porosity, fixed to $69 \%$, by:

$$
p_{S}^{\prime}\left(1-p_{S}\right)+p_{A} p_{S}=1-0.69 \text {. }
$$

An example of realization of a two-scales model is shown in Fig. (16). The volume of the microstructure is $400 \times 400 \times 546$ voxels at resolution $0.55 \mathrm{~nm}$ per voxel. The size of the $2 \mathrm{D}$ section is $400 \times 400$ pixels. The shape and size of the platelet is chosen the same as the prism shown in Fig. (1). The volume fraction of spheres $p_{S}$ is 0.5 , with sphere radius fixed at $8 \mathrm{~nm}$. The volume fraction of aligned platelets inside the spheres $p_{A}$ is 0.5 . In the generated microstructure, there are 5713 spheric aggregates, 165629 platelets aligned in the aggregates and 30364 randomly oriented platelets outside the aggregates. Local alignments are distinguished and encircled in red. We checked that the microstructure is macroscopicaly isotropic with respect to the correlation function. Hereafter, we let the 5 paremeters $D_{1,4}, p_{S, A}$ and $r_{S}$ vary. We optimize them to approach the TEM images' correlation function.

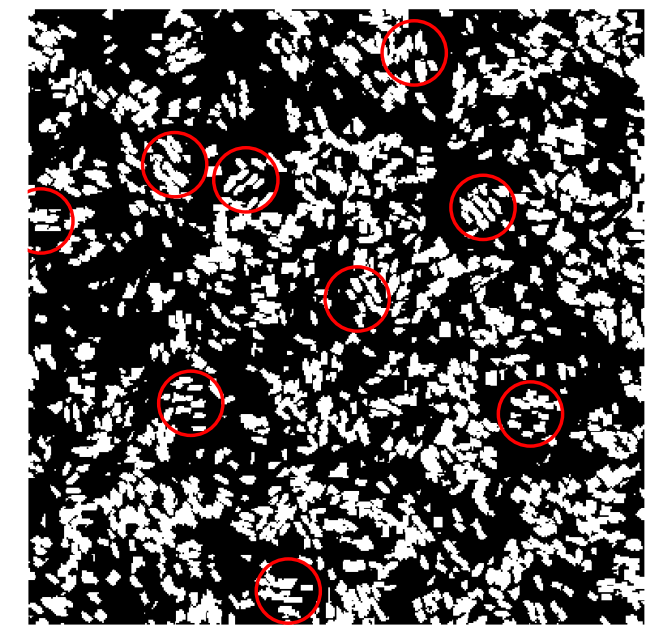

Figure 16: 2D section of a realization of the two-scales Boolean model with locally-aligned platelets (some of them encircled in red).

\subsection{Correlation function}

The effect of each parameter on the correlation function has firstly been examined numerically. Simulations are performed by fixing two of the three parameters, and letting the third parameter vary (Fig. 17). As expected, the slope at the origin of the correlation is not sensitive to the parameters governing large scale effects $p_{S}$ and $r_{S}$ (Figs. 17a and 17b). Their values however greatly affect the correlation range from $1.1 \mathrm{~nm}$ to $5.5 \mathrm{~nm}$. On the contrary, the slope at the origin of the correlation is sensitive to the volume fractions of platelets inside the grains $p_{A}$ (Fig. 17c), in particular for high values of $p_{A}$ larger than $50 \%$. In this regime, platelets often intersect, which greatly reduces their specific surface area. In the optimization process detailed below, the two parameters $p_{S}$ and $r_{S}$ are adjusted to control the correlation range, the other one being chosen to adjust the slope at the origin. 


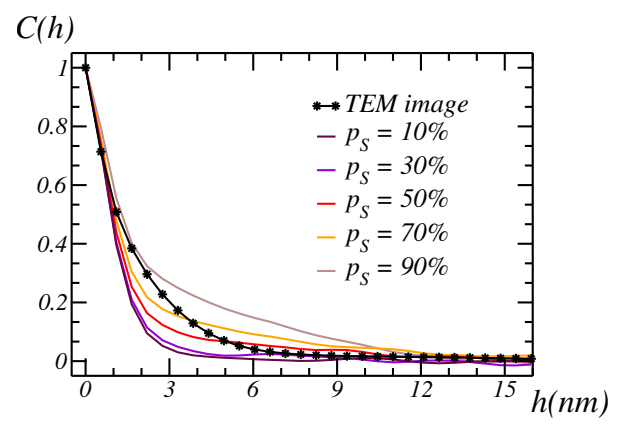

(a)

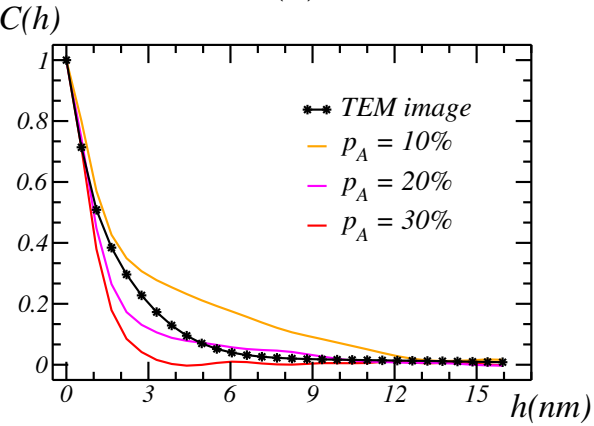

(c)

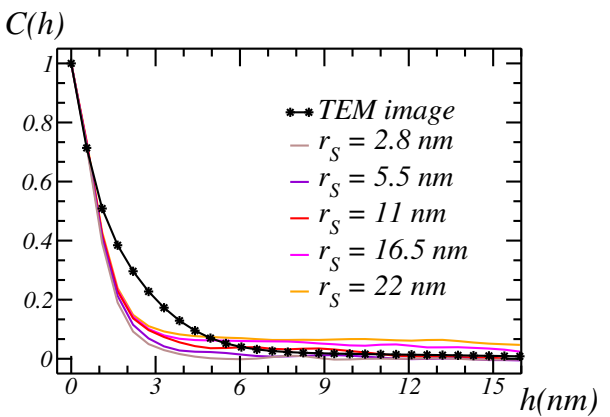

(b)

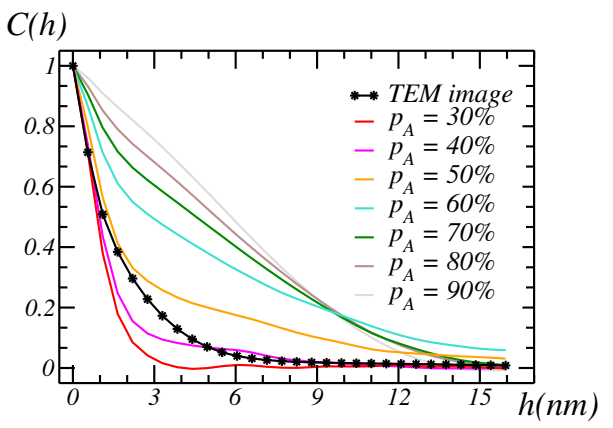

(d)

Figure 17: Effects of the spatial dispersion parameters $p_{S}, r_{S}$ and $p_{A}$ on the correlation function.

\subsection{Identification of the two-scales Boolean model}

Hereafter, the parameters of the two-scales Boolean model are estimated by optimization, to approach the correlation function of the TEM images. The same criterion is used as in the one-scale Boolean model (Eq. 10). First, a standard conjugate gradient descent method was tested. The method was ineffective, due to the high variability in the estimate of the gradient, especially for such two-scales model. The more robust Nelder-Mead method, which does not require the computation of a gradient (Nelder \& Mead, 1965), was also used. In most of our numerical simulations, the algorithm would very slowly converge. Furthermore, the microstructure was found to be sub-optimal. This is presumably an effect of variability.

Finally, a random "point cloud"simulations based on a Monte Carlo method (Hammersley \& Handscomb, 1964) was tested. To initialize the point cloud method, the shape parameters $D_{1}$ and $D_{4}$ are fixed, as in the optimized one-scale model. The cloud zone for the other parameters $\left(p_{A}, p_{S}\right.$ and $\left.r_{S}\right)$ is initially a large domain $\left(0.1<p_{A}<0.9\right.$, $0.1<p_{S}<0.9$ and $\left.2.6 \mathrm{~nm}<r_{s}<21 \mathrm{~nm}\right)$. In this domain, the specific surface area is not sensitive to $p_{A}$ and controlled by the values of $d_{A}$ and $d_{B}$ initially chosen. After random simulations, the zone of interest is reduced to a region where the objective function is lower. The process is repeated until convergence to a single point. The method, which tends to explore larger regions in the parameters space, was found to significantly reduce variability compared to the Nelder-Mead method, and also provided the best local minima. After 3 iterations, the algorithm would provide good-enough 
microstructures and was stopped.

The cloud optimization was repeated with varying shape parameters $D_{1}$ and $D_{4}$, following the specific surface area constraint (Eq. 12). A series of optimal two-scale models with respect to $D_{1}$ (or equivalently, $D_{4}$ ) is obtained. The correlation functions of two such models are represented in Fig. (18) and compared with that of the TEM images. For each set of parameters, the two correlation functions are in excellent agreement. We pick one of the two optimal models, corresponding to $D_{1}=3.3 \mathrm{~nm}$, and used in Fig. (18). This model is compared to the TEM image in Fig. (19). Image (19b) is simulated using (2) and a normalization similar to (3). For the sake of comparison, all values are comprised beteen 0 and 255. Note that the parameters $a, b$ and $c$ entering (2) are irrelevant due to (3).

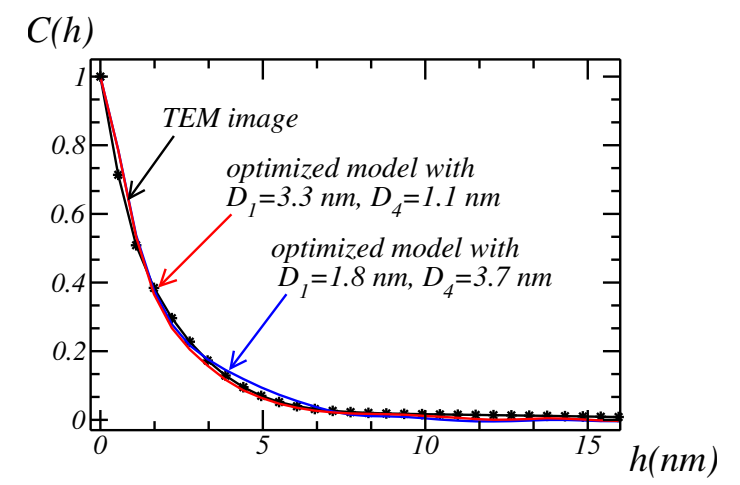

Figure 18: Correlation functions of two optimized two-scales models with different shape parameters for the grains: comparison with the correlation function of TEM images.

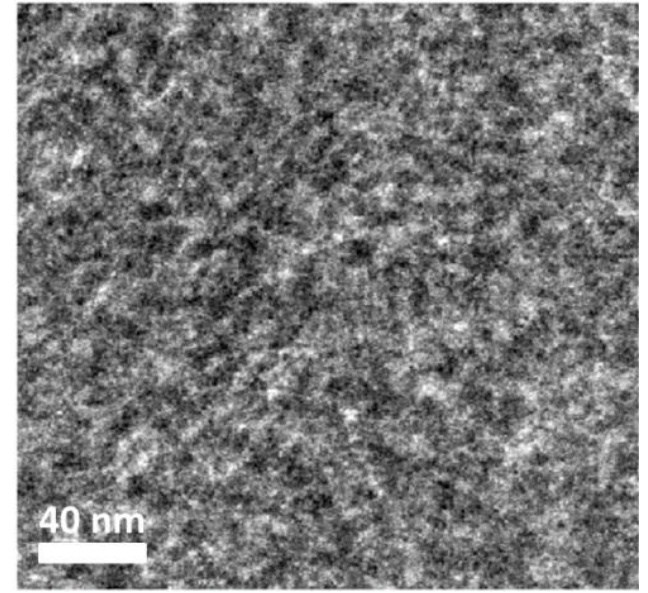

(a)

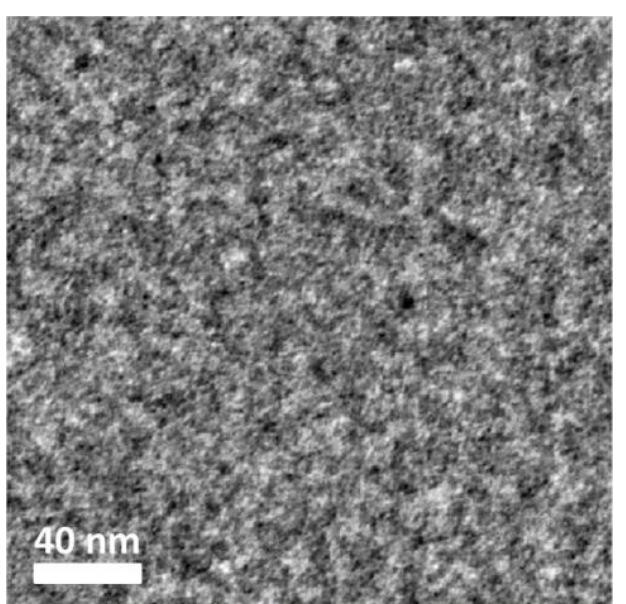

(b)

Figure 19: Comparison between (a) the TEM image and (b) the simulated transmission image using one of the optimal two-scales models with $D_{1}=3.3 \mathrm{~nm}$ (see Fig. 18). Image (b) is generated from Eq. (2) and a normalization similar to (3). 


\section{Porosimetry data and specific surface area}

In this section, the specific surface area of the optimized models and obtained from available porosimetry data are compared. Nitrogen porosimetry on the sample gives a specific surface area of $0.232 \mathrm{~nm}^{-1}$. For an arbitrary random set $A$, the specific surface area $S_{V}$ of $A$ is given by the slope at the origin of the correlation $Q(h)$, as reminded in equation 12. For two-scales models $B$ written as:

$$
B=\left(A_{0} \cap A_{1}\right) \cup\left(A_{0}^{c} \cap A_{2}\right),
$$

where $A_{i}(i=0,1,2)$ are independent random set with volume fraction $p_{i}$ and specific surface area $S_{V}^{(i)}$, and $A_{0}^{c}$ is the complementary of $A_{0}$, the specific surface area $S_{V}^{B}$ of the random set $B$ is given by (Jeulin, 2014):

$$
S_{V}^{B}=S_{V}^{(0)}\left(p_{1}+p_{2}-2 p_{1} p_{2}\right)+p_{0} S_{V}^{(1)}+\left(1-p_{0}\right) S_{V}^{(2)} .
$$

The first term in the right-hand side represents the specific surface of the set $B$ cut by that of $A_{0}$. The next terms are the specific surface in the interior and exterior of $A_{0}$. This equation is exact for platelets that are cut off at the boundary of primary grains. It can be applied to the two-scales Boolean model as follows:

$$
\begin{aligned}
& S_{V}^{(0)}=-\frac{3}{r_{S}}\left(1-p_{S}\right) \log \left(1-p_{S}\right), \quad S_{V}^{(1)}=-\frac{S_{p}}{V_{p}}\left(1-p_{A}\right) \log \left(1-p_{A}\right), \\
& S_{V}^{(2)}=-\frac{S_{p}}{V_{p}}\left(1-p_{S}^{\prime}\right) \log \left(1-p_{S}^{\prime}\right),
\end{aligned}
$$

where $\frac{S_{p}}{V_{p}}$ is the surface/volume ratio of the platelets, determined by $D_{1}$ and $D_{4}$ by simple geometric considerations. The resulting estimation for $S_{V}^{B}$ is only an approximation, since Eq. (13) is not exact for the Cox Boolean model. However, the approximation is correct if a large scale-separation $r_{S} \gg D_{1}$ and $r_{S} \gg D_{4}$ is assumed (Jeulin, 2012). In the present two-scales model, $r_{S} / D_{1}$ and $r_{S} / D_{4}$ are at least equal to 2 .

In a first step, a particular optimized two-scales microstructure with $D_{1}=3.3 \mathrm{~nm}$ and $D_{4}=1.1 \mathrm{~nm}$ (corresponding to Fig. 18) is considered. The porosimetry results depend on the size of the molecules used for adsorption experiments (Thommes et al., 2000; Wernert et al., 2010). The estimated specific surface areas are higher (resp. lower) when small (resp. large) molecules are used. This effect is especially important in nanomaterials. To roughly model the nitrogen adsorption, morphological closings (dilation followed by erosion) by a cube, a 3D cross and a rhombicuboctahedron of increasing sizes are first performed. The rhombicuboctahedron better approximates the shape of molecules on a digitized image, and offers a good compromise between performance and exactness. Its size corresponds to the radius of an equivalent sphere. Second, closed pores that are not accessible to nitrogen are removed. 3D views of one optimized two-scales microstructure after closing are represented in Fig. (20). Results for the estimates of the specific surface areas are given in Tab. (2). We denote by $S_{V}^{\text {cov }}$ the estimate of the slope of the tangent line to the correlation function at $h=0$ (Eq. 12). For comparison purposes, we also give estimates obtained by the method of weighted local configurations proposed in (Ziegel \& Kiderlen, 2010) and analytical 
estimates from Eq. (14), denoted $S_{V}^{Z}$ and $S_{V}^{B}$ respectively. The method in (Ziegel \& Kiderlen, 2010) makes use of 5 weights which depend on two parameters $s$ and $r$. We follow (Ziegel \& Kiderlen, 2010) and set $s=1.7452$ and $r=1$. These two values are given for cylinders. They give nearly optimal estimates with the platelets used in the present work.

The estimates $S_{V}^{\text {cov }}, S_{V}^{Z}$ are measured on the original microstructure $(M)$, its closing by a cube $\left(M^{\prime}\right)$ and by a $3 \mathrm{D}$ cross $\left(M^{\prime \prime}\right)$ of size 1 pixel $(0.55 \mathrm{~nm})$. We also remove closed pores by a hole filling operation on the three microstructures and estimate their specific surface area $S_{V}^{Z^{\prime}}$, using the method of Ziegel.

Numerical results for the specific surface are of the same order than experimental ones (about $0.232 \mathrm{~nm}^{-1}$ ). Closing also significantly influences the specific surface area. Furthermore, numerical results indicate that the latter is affected by the shape of the structuring element. There are very few closed pores in the microstructure, as indicated by the values of $S_{V}^{Z^{\prime}}$, very close to $S_{V}^{Z}$.

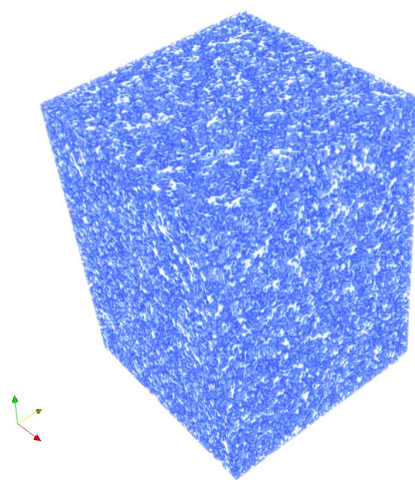

$\delta=0$

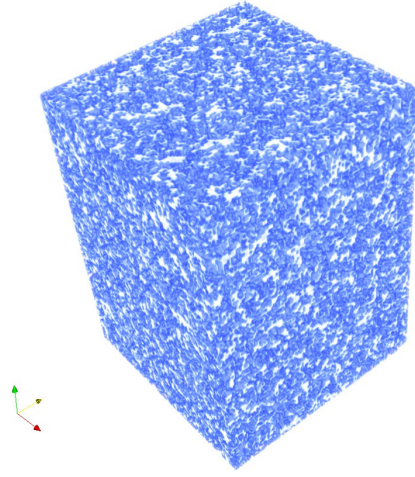

$\delta=1$

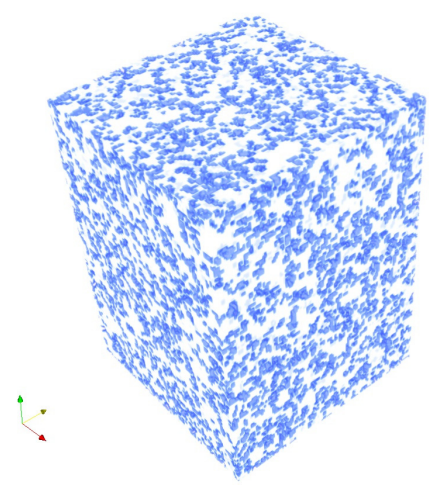

$\delta=2$

Figure 20: 3D views of the closings of an optimized two-scales Boolean model with increasing structuring element size (left to right). Porosity in blue and platelets in white. The structuring element is a rhombicuboctahedron of size $\delta$ (in voxels).

\begin{tabular}{|l||l|l|l|l|}
\hline & $S_{V}^{B}\left(\mathrm{~nm}^{-1}\right)$ & $S_{V}^{\text {cov }}\left(\mathrm{nm}^{-1}\right)$ & $S_{V}^{Z}\left(\mathrm{~nm}^{-1}\right)$ & $S_{V}^{Z^{\prime}}\left(\mathrm{nm}^{-1}\right)$ \\
\hline$M$ & 0.8104 & 0.6611 & 0.5533 & 0.5527 \\
\hline$M^{\prime}$ & & 0.4225 & 0.4299 & 0.4277 \\
\hline$M^{\prime \prime}$ & & 0.5796 & 0.4878 & 0.4871 \\
\hline
\end{tabular}

Table 2: Specific surface areas of the two-scales optimized Boolean model $M$, and of its closing $M^{\prime}$ and $M^{\prime \prime}$, computed using the analytical estimate (Eq. 14), the correlation function and the method of weighted local configurations in (Ziegel \& Kiderlen, 2010). The analytical estimate (Eq. 14) is relevant for model $M$ only. 


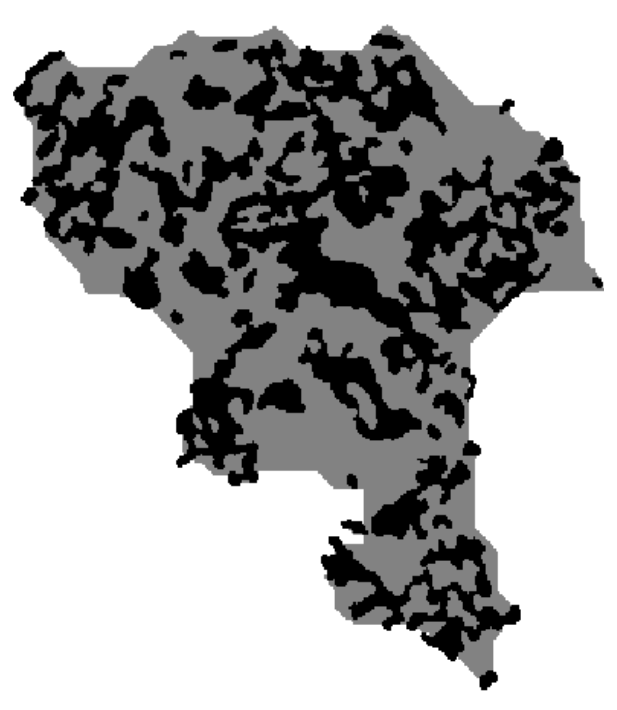

Figure 21: Porous volume of the tomography image after closing with rhombicuboctahedron of 30 voxels and erosion by 3 voxels (2D section). Alumina grains are in dark, while in grey is the porosity.

\subsection{Comparison with the tomographic model}

Hereafter, the two-scales models of platelets are compared to the tomographic model in terms of porosimetry. The porous volume of the tomographic model is extracted by using a series of morphological operations proposed by Moreaud et al (Moreaud et al., 2008). First, a closing operation by a sufficient size (here we take 30 voxels) together with a hole filling operation is performed to remove all pores in its interior. Next, a geodesic erosion is performed on the 3D image in order to maintain the surface irregularity (Fig. 21). The global volume and the external surface contour of the tomographic model are emerged after the erosion.

Then, closings of increasing sizes are performed with rhombicuboctahedra on both the tomographic model and the two-scales microstructure models, to simulate the nitrogen adsorption. The structuring element roughly mimics the role of nitrogen molecules. Isolated pores are removed using hole filling operations to extract the accessible porosity. Specific surface area measurement results are given in Fig. (22), in red for the area of accessible specific surface in the two-scales model, and in blue for the area of accessible specific surface in the tomographic model. These values have been corrected by subtracting the exterior surface area of the specimen. Finally, the nitrogen porosimetry data $\left(0.231 \mathrm{~nm}^{-1}\right)$ is indicated by the dotted black horizontal lines. The nitrogen molecules whose shapes are anisotropic are indicated by vertical black dotted lines.

As shown in Fig. (22), the specific surface area in the two-scales microstructure agrees with porosimetry data when the size of the structuring element is about $1.2 \mathrm{~nm}$. This value is quite higher than the nitrogen size, of about 0.3 and $0.4 \mathrm{~nm}$. For a structuring element of this size, the estimated specific surface area is about 2 to 3 times higher for the two-scales model compared to porosimetry data and to the tomographic model. However, the difference between model and porosimetry data is much less of the 


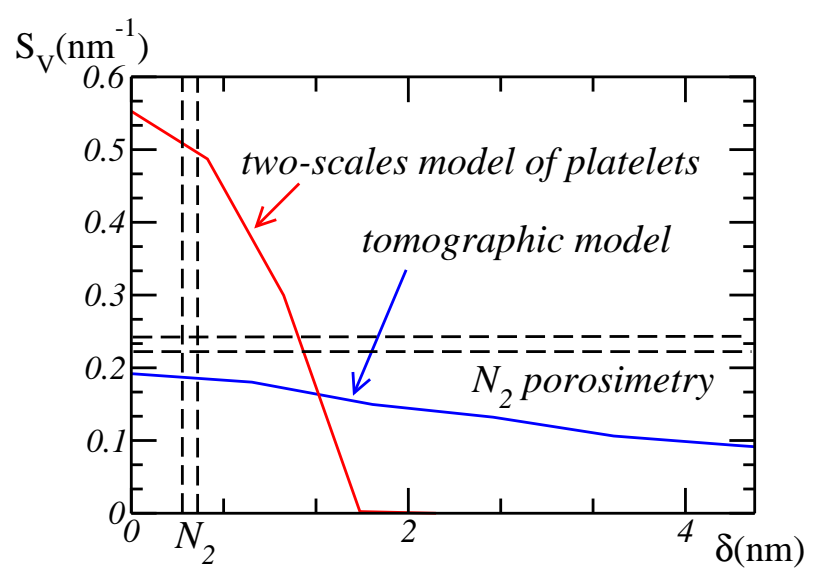

Figure 22: Specific surface areas with respect to the size of the closing: comparison between one two-scales model of platelets (red), the tomographic model (blue) and porosimetry data in confidence interval (horizontal black dotted lines). The sizes of nitrogen molecules are indicated by the two vertical black dotted lines.

same order than usually observed when comparing image analysis results to molecular porosimetry.

Some differences between the two-scales optimized Boolean model and tomographic image are also observed. The decrease of the surface density with respect to the size of the structuring element is much steeper in the two-scales model than in the tomographic image. The difference between accessible and overall properties is also more important in the two-scales model, for large structuring elements.

Finally, the accessible porosity globally follows the same trend as the specific surface area when the size of the structuring element increases (Fig. 23). Also, our results are nearly insensitive to the shape of the platelets in the two-scales model. Nearly identical results have been obtained when considering other two-scales optimized models with varying shape parameters (not shown).

\section{Conclusion}

In this work, a two-scales Boolean microstructure was proposed to model mesoporous alumina. The model is based on transmission images where correlation lengths are quite larger than that of the observed platelets in the material. The model takes into account the spatial dispersion of platelets in the material, making extensive use of the correlation function. The latter is quite insensitive to the exact platelet shape, which could not be accurately determined from the available noisy transmission images. Nevertheless, the model supports a range of physically-admissible platelets shapes, as deduced from Xray diffraction measurements. The estimation of the five parameters of the model was carried out by optimization, using a random point cloud process, which was found to be more robust than the Nelder-Mead and conjugate gradient methods. Furthermore, the optimization was constrained by decoupling the effect of the shape parameters for 


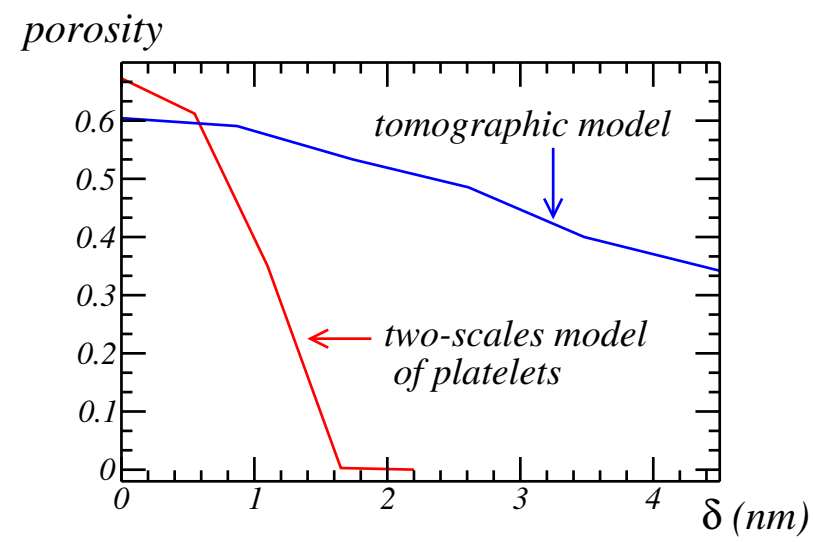

Figure 23: Accessible porosity $V_{V}$ after closings by rhombicuboctahedra of increasing size, for the two-scales optimized and tomographic models (red and blue, resp.).

the platelets, which control the slope of the correlation at the origin, and the other 3 parameters which affect the dispersion at larger scales.

The model was compared with a 3D tomographic reconstruction of a small-size part of the material. Microstructural properties, in terms of specific surface area, were compared with measurements on tomographic images and with data provided by nitrogen porosimetry and density. These measurements differ quantitatively, but are of the same order. Part of this difference is explained by the amount of porosity accessible to nitrogen particles in porosimetry experiments. Further experimental and modelling works are needed to clarify the observed discrepancies.

Results obtained in this work indicate that hierarchical or multiscale models are presumably necessary to reproduce quantitatively the transport and sorption properties of mesoporous alumina, particularly the nitrogen isotherms. Future study will focus on the influences of different morphologies of microstructure on the transport properties, i.e. nitrogen isotherms of mesoporous alumina. This approach can be applied to further nanoporous materials.

\section{References}

Adler, P.M. (1994) The method of reconstructed porous media. Phys. Fluids 1, 227.

Altendorf, H., Jeulin, D. \& Willot, F. (2014) Influence of the fiber geometry on the macroscopic elastic and thermal properties. Int. J. Sol. Struct. 51, 3807-3822.

Brunauer, S., Emmett, P.H. \& Teller, E. (1938) Adsorption of gases in multimolecular layers. J. Am. Chem. Soc. 60, 309-319.

Couka, E., Willot, F., Jeulin, D., Achour, M.B., Chesnaud, A. \& Thorel, A. (2015) Modeling of the multiscale dispersion of nanoparticles in a hematite coating. $J$. Nanosci. Nanotechnol. 15(5), 3515-3521. 
Chiche, D., Digne, M., Revel, R., Chaneac, C. \& Jolivet, J. (2008) Accurate determination of oxide nanoparticle size and shape based on x-ray powder pattern simulation: application to boehmite AlOOH. J. Phys. Chem. C 112, 8524-8533.

Diaz, I., Gonzalez-Pena, V., Marquez-Alvarez, C. \& Kikkinides, E.S. (2004) Transmission electron microscopy combined with stochastic reconstruction methods for structural characterization of porous alumina synthesized via non-ionic surfactanttemplating route. Microporous Mesoporous Mater. 68, 11-19.

Digne, M., Sautet, P., Raybaud, P., Euzen, P. \& Toulhoat, H. (2004) Use of DFT to achieve a rational understanding of acid-basic properties of $\gamma$-alumina surfaces. $J$. Catal. 226, 54-68.

Euzen, P., Raybaud, P., Krokidis, X., Toulhoat, H., Le Loarer, J.-L., Jolivet, J.-P. \& Froidefond, C. (2002) Alumina. Handbook of Porous Solids (ed. by Schüth, F., Sing, K. \& Weitkamp, J.), Wiley-VCH Verlag GmbH, Weinheim, Germany.

Fukusawa, J. \& Tsujii, K. (1988) Higher-order structure formation of ultrafine boehmite particles in sols, gels, and dried materials. J. Colloid Interface Sci. 125, 155-161.

Hammersley, J. \& Handscomb, D. (1964) Monte carlo methods. Methuen, London.

Jean, A., Jeulin, D., Forest, S., Cantournet, S. \& N'Guyenm, F. (2010) A multiscale microstructure model of carbon black distribution in rubber. J. Microsc. 241, 243260.

Jeulin, D. (2000) Random texture models for material structures. Stat. Comput. 10, $121-132$.

Jeulin, D. (2011) Variance scaling of Boolean random varieties. <hal-00618967>.

Jeulin, D. (2012) Morphology and effective properties of multi-scale random sets: A review. C. R. Mecanique, 340, 219-229.

Jeulin, D. (2013) Correlation of orientations in a vector field and statistics of alignments. Internal Report, CMM, Mines ParisTech, N-02/13/MM.

Jeulin, D. (2014) A two-scales platelet random model. Internal Report, CMM, Mines ParisTech, N-02/14/MM.

Kanit, T., Forest, S., Galliet, I., Mounoury, V. \& Jeulin, D. (2003) Determination of the size of the representative volume element for random composites: statistical and numerical approach. Int. J. Solids. Struct. 40, 3647-3679.

Khodakov, A.Y., Griboval-Constant, A., Bechara, R. \& Zholobenko, V. (2002) Pore size effects in Fischer Tropsch synthesis over Cobalt-supported mesoporous silicas. J. Catal. 206, 230-241. 
Kim, P., Kim, Y., Kim, C., Kim, H., Park, Y., Lee, J.H., Song, I.K. \& Yi, J. (2003) Synthesis and characterization of mesoporous alumina as a catalyst support for hydrodechlorination of 1,2-dichloropropane: effect of catalyst preparation method. Catal. Lett. 89, 3-4.

Kočí, P., Štěpanék, F., Kubíček, M. \& Marek, M. (2006) Meso-scale modelling of CO oxidation in digitally reconstructed porous $\mathrm{Pt} / \gamma-\mathrm{Al}_{2} \mathrm{O}_{3}$ catalyst. Chem. Eng. Sci. 61, 3240-3249.

Kočí, P., Štěpanék, F., Kubíček, M. \& Marek, M. (2007) Pore-scale modeling of non-isothermal reaction phenomena in digitally reconstructed porous catalyst. Mol. Simul. 33(4-5), 369-377.

Kohout, M., Collier, A.P. \& Štěpanék, F. (2004) Effective thermal conductivity of wet particle assemblies. Int. J. Heat. Mass. Tran. 47(25), 5565-5574.

Lantuéjoul, C. (1991) Ergodicity and integral range. J. Microsc. 161, 387-403.

Levitz, P. (1993) Knudsen diffusion and excitation transfer in random porous media. J. Phys. Chem. 97, 3813-3818.

Matheron, G. (1967) Eléments pour une théorie des milieux poreux. Masson, Paris.

Matheron, G. (1971) The theory of regionalized variables and its applications. École Nationale Supérieure des Mines de Paris, Fontainebleau.

Moreaud, M., Celse, B. \& Tihay, F. (2008) Analysis of the accessibility of macroporous alumino-silicate using 3D-TEM. Materials Science $\mathcal{E}$ Technology, October 5-9, Pittsburgh.

Misra, C. (1987) Industrial Alumina Chemicals. Anal. Chem., 59(10), 706A-706A.

Moreaud, M., Jeulin, D., Morard, V. \& Revel, R. (2012) TEM image analysis and modelling: application to boehmite nanoparticles. J. Microsc. 245, 186-199.

Moreland, K., Avila, L. \& Fisk, L.A. (2007) Parallel unstructured volume rendering in ParaView. Proceedings of ISEST SPIE Visualization and Data Analysis 200\%, San Jose, CA.

Mu, D., Liu, Z., Huang, C. \& Djilali, N. (2008) Determination of the effective diffusion coefficient in porous media including Knudsen effects. Microfluid Nanofluidics 4, $257-260$.

Nelder, J. \& Mead, R. (1965) A simplex method for function minimization. Comput. J. 7, 308-313.

Prieto, G., Shakeri, M., de Jong, K.P. \& de Jongh, P.E. (2014) Quantitative relationship between support porosity and the stability of pore-confined metal nanoparticles studied on $\mathrm{CuZnO} / \mathrm{SiO}_{2}$ methanol synthesis catalysts. ACS Nano 8(3), 2522-2531. 
Rana, M.S., Ancheyta, J., Maity, S.K. \& Rayo, P. (2011) Hydrotreating of Maya crude oil: I. effect of support composition and its pore-diameter on asphaltene conversion. Pet. Sci. Technol. 25(1-2), 187-199.

Reimer, L. \& Kohl, H. (2008) Transmission electron microscopy: physics of image formation. Springer, New York.

Roiban, L. (2010) Tomographie electronique analytique: developpement de la cartographie chimique 3D a l'echelle du nanometre et application aux catalyseurs heterogenes. PhD thesis, Strasbourg University, Strasbourg.

Štěpanék, F., Soos, M. \& Rajniak, P. (2007) Characterization of porous media by the virtual capillary condensation method. Colloids Surf., A 300, 11-20.

Thommes, M., Kohn, R. \& Froba, M. (2000) Sorption and pore condensation behavior of nitrogen, argon and krypton in mesoporous MCM-48 silica materials. J. Phys. Chem. B 104, 7932-7943.

Tran, V.D., Moreaud, M., Thiebault, E., Denis, L. \& Becker, J.M. (2014) Inverse problem approach for the alignment of electron tomographic series. Oil Gas Sci. Technol. 69(2), 279-291.

Trimm, D.L. \& Stanislaus, A. (1986) The control of pore size in alumina catalyst supports: a review. Appl. Catal. 21, 215-238.

Wernert, V., Bouchet, R. \& Denoyel, R. (2010) Influence of molecule size on its transport properties through a porous medium. Anal. Chem. 82, 2668-2679.

Ziegel, J. \& Kiderlen, M. (2010) Estimation of surface area and surface area measure of three-dimensional sets from digitizations. Image Vision Comput. 28, 64-77. 